\title{
On the Stochastic Stability and Boundedness of Solutions for Stochastic Delay Differential Equation of the Second Order
}

\author{
A. M. A. Abou-El-Ela, ${ }^{1}$ A. I. Sadek, ${ }^{1}$ A. M. Mahmoud, ${ }^{2}$ and R. O. A. Taie ${ }^{1}$ \\ ${ }^{1}$ Department of Mathematics, Faculty of Science, Assiut University, Assiut 71516, Egypt \\ ${ }^{2}$ Department of Mathematics, Faculty of Science, Assiut University, New Valley Branch, Al Khargah, New Valley 72111, Egypt
}

Correspondence should be addressed to R. O. A. Taie; rasha_omath@yahoo.com

Received 23 November 2014; Revised 18 March 2015; Accepted 19 March 2015

Academic Editor: Zhen-Lai Han

Copyright (C) 2015 A. M. A. Abou-El-Ela et al. This is an open access article distributed under the Creative Commons Attribution License, which permits unrestricted use, distribution, and reproduction in any medium, provided the original work is properly cited.

\begin{abstract}
We present two qualitative results concerning the solutions of the following equation: $\ddot{x}(t)+g(\dot{x}(t))+b x(t-h)+\sigma x(t) \dot{\omega}(t)=$ $p(t, x(t), \dot{x}(t), x(t-h))$; the first result covers the stochastic asymptotic stability of the zero solution for the above equation in case $p \equiv 0$, while the second one discusses the uniform stochastic boundedness of all solutions in case $p \not \equiv 0$. Sufficient conditions for the stability and boundedness of solutions for the considered equation are obtained by constructing a Lyapunov functional. Two examples are also discussed to illustrate the efficiency of the obtained results.
\end{abstract}

\section{Introduction}

During the last twenty years, the theory of stochastic differential equations has successfully attracted considerable attentions of scholars; for example, see [1-13]. Since then, the number of contributions to statistics, numerics, and control theory of stochastic differential equations has been rapidly increasing, since stochastic modelling plays an important role in formulation and analysis in modelling physical, technical, biological and economical dynamical systems in which significant uncertainty is present.

Stochastic delay differential equation, also known as stochastic functional differential equation, is a natural generalization of stochastic ordinary differential equation by allowing the coefficients to depend on the past values. The Razumikhin argument, generalized Itô formula, and Euler-Maruyama formula play an important role in studying stochastic differential equations. Unfortunately, it is generally not possible to give explicit expressions for the solutions to stochastic differential equations. Therefore, most of the papers are interested in being able to characterize at least qualitatively the behaviour of the solutions. Thus, Lyapunov theory is a powerful tool for qualitative analysis of stochastic differential equations, since the advantage of this method can judge the behaviour of systems without any prior knowledge of the explicit solutions, while the greatest disadvantage of the Lyapunov approach is that no universal method has been given, which enables us to find a Lyapunov function or determine that no such function exists.

It is worth mentioning that there are few results on the stability and boundedness of solutions for first-order stochastic delay differential equations; for example, see [8, 14-18].

In 2004, Kolmanovskii and Shaikhet [8] investigated the conditions of asymptotic mean-square stability for first-order stochastic delay differential equation of neutral type:

$$
\begin{aligned}
& \dot{x}(t)+a x(t)+b x(t-h)+c \dot{x}(t-h)+\sigma x(t-\tau) \dot{\xi}(t)=0, \\
&|c|<1,
\end{aligned}
$$

where $a$ and $b$ are two positive constants and $\xi(t)$ is a standard Wiener process.

Later, in 2006, Rodkina and Basin [17] obtained global asymptotic stability conditions for nonlinear stochastic systems with state delay as follows:

$$
\begin{aligned}
& d x(t)=-a N(x(t)) d t-b N(x(t-h)) d t+\sigma\left(t, x_{t}\right) d w_{t}, \\
& x(s)=\phi(s), \quad s \in[-h, 0] .
\end{aligned}
$$


The Lyapunov Krasovskii and degenerate functionals techniques are used. In addition, nontrivial examples of nonlinear systems satisfying the obtained stability conditions are given.

On the other hand, the corresponding problem for the stability and boundedness of solutions of second-order stochastic delay differential equations was studied far less often. So our main aim in this paper is to establish new results on the stability and boundedness for solutions of secondorder stochastic delay differential equation of the type

$$
\begin{aligned}
\ddot{x}(t) & +g(\dot{x}(t))+b x(t-h)+\sigma x(t) \dot{\omega}(t) \\
= & p(t, x(t), \dot{x}(t), x(t-h)),
\end{aligned}
$$

where $b$ and $\sigma$ are two positive constants, and $h$ is a positive constant delay; $g$ and $p$ are two continuous functions with $g(0)=0 ; \omega(t)=\left(\omega_{1}(t), \omega_{2}(t), \ldots, \omega_{m}(t)\right) \in \mathbb{R}^{m}$ is an $m$-dimensional standard Brownian motion defined on the probability space (also called Wiener process), a stochastic process representing the noise [19].

\section{Preliminaries and Stability Result}

Consider the following nonautonomous $n$-dimensional stochastic delay differential equation (SDDE):

$$
\begin{aligned}
d x(t)= & f(t, x(t), x(t-\tau)) d t \\
& +g(t, x(t), x(t-\tau)) d B(t), \quad t \geq 0,
\end{aligned}
$$

where $f: \mathbb{R}^{+} \times \mathbb{R}^{n} \times \mathbb{R}^{n} \rightarrow \mathbb{R}^{n}$ and $g: \mathbb{R}^{+} \times \mathbb{R}^{n} \times$ $\mathbb{R}^{n} \rightarrow \mathbb{R}^{n \times m}$ are given nonlinear continuous functions, $B(t)=\left(B_{1}(t), B_{2}(t), \ldots, B_{m}(t)\right)$ is an $m$-dimensional standard Brownian motion, and $x(t)=\left(x_{1}(t), x_{2}(t), \ldots, x_{n}(t)\right) \in \mathbb{R}^{n}$ is a solution of the stochastic delay differential equation (4) with initial data of $\{x(\theta):-\tau \leq \theta \leq 0\}, x_{0} \in \mathscr{C}\left([-\tau, 0] ; \mathbb{R}^{n}\right)$. We assume that $f$ and $g$ satisfy the following conditions.

Standing Hypothesis (H1). Both $f$ and $g$ satisfy the local Lipschitz condition and for all given $b>0, p \geq 2, f(t, 0,0) \in$ $\mathscr{L}^{1}\left([0, b] ; \mathbb{R}^{n}\right)$, and $g(t, 0,0) \in \mathscr{L}^{p}\left([0, b] ; \mathbb{R}^{n \times m}\right)$ (see $\left.[20]\right)$. Then, there must be a stopping time $\beta=\beta(\omega)>0$ such that (4) with $x_{0} \in \mathscr{C}_{\mathscr{F}_{0}}^{p}$ has a unique maximal local solution for $t \in\left[t_{0}, \beta\right)$.

In this section, for the stability result, we impose Standing Hypothesis (H2):

$$
f(t, 0,0)=0, \quad g(t, 0,0)=0, \quad \forall t \geq 0 .
$$

Hence, the stochastic delay differential equation (4) admits the zero solution $x(t ; 0) \equiv 0$, for any given initial value $x_{0} \in \mathscr{C}\left([-\tau, 0] ; \mathbb{R}^{n}\right)$.

Definition 1. The zero solution of the stochastic differential equation is said to be stochastically stable or stable in probability, if for every pair of $\epsilon \in(0,1)$ and $r>0$, there exists a $\delta=\delta(\epsilon, r)>0$ such that

$$
P\left\{\left|x\left(t ; x_{0}\right)\right|<r \forall t \geq 0\right\} \geq 1-\epsilon, \quad \text { whenever }\left|x_{0}\right|<\delta .
$$

Otherwise, it is said to be stochastically unstable.
Definition 2. The zero solution of the stochastic differential equation is said to be stochastically asymptotically stable, if it is stochastically stable, and moreover for every $\epsilon \in(0,1)$, there exists a $\delta_{0}=\delta_{0}(\epsilon)>0$ such that

$$
P\left\{\lim _{t \rightarrow 0} x\left(t ; x_{0}\right)=0\right\} \geq 1-\epsilon, \quad \text { whenever }\left|x_{0}\right|<\delta_{0} .
$$

Let $\mathscr{K}$ denote the family of all continuous nondecreasing functions $\rho: \mathbb{R}^{+} \rightarrow \mathbb{R}^{+}$such that $\rho(0)=0$ and $\rho(r)>0$, if $r>0$. In addition, $\mathscr{K}_{\infty}$ denotes the family of all functions $\rho \in \mathscr{K}$ with $\lim _{r \rightarrow \infty} \rho(r)=\infty$. Let $\mathscr{C}^{1,2}\left(\mathbb{R}^{+} \times \mathbb{R}^{n} ; \mathbb{R}^{+}\right)$ denote the family of nonnegative functions $V(t, x)$ defined on $\mathbb{R}^{+} \times \mathbb{R}^{n}$ which are once continuously differentiable in $t$ and twice continuously differentiable in $x$. For each $V \in \mathscr{C}^{1,2}\left(\mathbb{R}^{+} \times\right.$ $\mathbb{R}^{n} ; \mathbb{R}^{+}$), define an operator $\mathscr{L} V: \mathbb{R}^{+} \times \mathbb{R}^{n} \times \mathbb{R}^{n} \rightarrow \mathbb{R}$ by

$$
\begin{aligned}
\mathscr{L} V(t, x, y)= & V_{t}(t, x)+V_{x}(t, x) f(t, x, y) \\
& +\frac{1}{2} \operatorname{trace}\left[g^{T}(t, x, y) V_{x x}(t, x) g(t, x, y)\right],
\end{aligned}
$$

where

$$
\begin{aligned}
V_{t}(t, x) & =\frac{\partial V(t, x)}{\partial t}, \\
V_{x}(t, x) & =\left(\frac{\partial V(t, x)}{\partial x_{1}}, \ldots, \frac{\partial V(t, x)}{\partial x_{n}}\right), \\
V_{x x}(t, x) & =\left(\frac{\partial^{2} V(t, x)}{\partial x_{i} \partial x_{j}}\right)_{n \times n} .
\end{aligned}
$$

It should be emphasized that the operator $\mathscr{L} \mathrm{V}$ is defined on $\mathbb{R}^{+} \times \mathbb{R}^{n} \times \mathbb{R}^{n}$, although the function $V$ is defined only on $\mathbb{R}^{+} \times \mathbb{R}^{n}$

To prove our main stability result, we shall introduce the following theorems.

Theorem 3 (see $[21,22])$. Assume that there exist $V \in$ $\mathscr{C}^{1,2}\left(\mathbb{R}^{+} \times \mathbb{R}^{n} ; \mathbb{R}^{+}\right)$and $\mu \in \mathscr{K}_{\infty}$ such that

$$
\begin{array}{r}
\mu(|x|) \leq V(t, x), \quad \mathscr{L} V(t, x, y) \leq 0, \\
\forall(t ; x ; y) \in \mathbb{R}^{+} \times \mathbb{R}^{n} \times \mathbb{R}^{n} .
\end{array}
$$

Then, (4) with $x_{0} \in \mathscr{C}_{\mathscr{F}_{0}}^{p}$ has a unique global solution for $t>0$ denoted by $x\left(t ; t_{0}\right)$ (this is a special case of Corollary 12 in [22] with $G \equiv b \equiv \kappa \equiv 0)$.

Furthermore, $V(0)=0$; then, the zero solution of the stochastic delay differential equation is stochastically stable (see [21]).

Theorem 4 (see [21]). Assume that there exist $V \in \mathscr{C}^{1,2}\left(\mathbb{R}^{+} \times\right.$ $\left.\mathbb{R}^{n} ; \mathbb{R}^{+}\right)$and $\mu_{1}, \mu_{2}, \mu_{3} \in \mathscr{K}$ such that

$$
\begin{gathered}
\mu_{1}(|x|) \leq V(t, x) \leq \mu_{2}(|x|), \\
\mathscr{L} V(t, x, y) \leq-\mu_{3}(|x|),
\end{gathered}
$$

for all $(t, x, y) \in \mathbb{R}^{+} \times \mathbb{R}^{n} \times \mathbb{R}^{n}$. Then, the zero solution of the stochastic delay differential equation is stochastically asymptotically stable.

Now we present the main stability result of (3) with $p \equiv 0$. 
Theorem 5. Further to the basic assumptions imposed on a, $b, \sigma, h, g$, and $p$, suppose that the following conditions are satisfied.

(i) $g(y) / y \geq a>0$ for $y \neq 0$ and $\left|g^{\prime}(y)\right| \leq l, l>0$, for all $y$.

(ii) $a>b>1$.

(iii) $b(a-1)-(l+1) \sigma^{2}>0$.

Then, the zero solution of (3) is stochastically asymptotically stable, provided that

$$
h<\min \left\{\frac{b(a-1)-(l+1) \sigma^{2}}{2 a b}, \frac{a(a-b)+a^{2}-b}{2 b(a+2 l+2)}\right\} .
$$

Proof of Theorem 5. In fact, (3) with $p \equiv 0$ can be transformed into an equivalent system of the following form:

$$
\begin{aligned}
& \dot{x}=y \\
& \dot{y}=-g(y)-b x+b \int_{t-h}^{t} y(s) d s-\sigma x \dot{\omega}(t) .
\end{aligned}
$$

Define the Lyapunov functional $V_{1}\left(x_{t}, y_{t}\right)$ as

$$
\begin{aligned}
V_{1}\left(x_{t}, y_{t}\right)= & \int_{0}^{y} g(\eta) d \eta+b x^{2}+\frac{1}{2}(y+a x)^{2} \\
& +\gamma \int_{-h}^{0} \int_{t+s}^{t} y^{2}(\phi) d \phi d s,
\end{aligned}
$$

where $x_{t}=x(t+s), s \leq 0$, and $\gamma$ is a positive constant, which will be determined later.

From (14) and (13) and by using Itô formula, we find

$$
\begin{aligned}
\mathscr{L} V_{1}\left(x_{t}, y_{t}\right)= & a y^{2}+a^{2} x y-g^{2}(y)-b x g(y) \\
& +b g(y) \int_{t-h}^{t} y(s) d s-y g(y) \\
& +b x y+b y \int_{t-h}^{t} y(s) d s-a x g(y) \\
& -a b x^{2}+a b x \int_{t-h}^{t} y(s) d s+\gamma h y^{2}(t) \\
& -\gamma \int_{t-h}^{t} y^{2}(s) d s+\frac{g^{\prime}(y)+1}{2} \sigma^{2} x^{2} ;
\end{aligned}
$$

since $\left|g^{\prime}(y)\right| \leq l, l>0$ and $g(y) / y \geq a>0$ by (i), then

$$
\begin{aligned}
\mathscr{L} V_{1}\left(x_{t}, y_{t}\right) \leq & -a^{2} y^{2}-a b x y+b l y \int_{t-h}^{t} y(s) d s \\
& +b x y+b y \int_{t-h}^{t} y(s) d s-a b x^{2} \\
& +a b x \int_{t-h}^{t} y(s) d s+\gamma h y^{2} \\
& -\gamma \int_{t-h}^{t} y^{2}(s) d s+\frac{l+1}{2} \sigma^{2} x^{2} .
\end{aligned}
$$

Thus, by using the inequality $|u v| \leq(1 / 2)\left(u^{2}+v^{2}\right)$, we obtain

$$
\begin{aligned}
\mathscr{L} V_{1}\left(x_{t}, y_{t}\right) \leq & -\left\{\frac{b(a-1)}{2}-\frac{l+1}{2} \sigma^{2}-\frac{a b h}{2}\right\} x^{2} \\
& -\left\{\frac{a(a-b)+a^{2}-b}{2}-\left(\frac{b l}{2}+\frac{b}{2}+\gamma\right) h\right\} y^{2} \\
& +\left(\frac{b l}{2}+\frac{a b}{2}+\frac{b}{2}-\gamma\right) \int_{t-h}^{t} y^{2}(s) d s .
\end{aligned}
$$

Let us choose $\gamma=b(l+a+1) / 2>0$. Then, it is easy to see that

$$
\begin{aligned}
\mathscr{L} V_{1}\left(x_{t}, y_{t}\right) \leq & -\left\{\frac{b(a-1)}{2}-\frac{l+1}{2} \sigma^{2}-\frac{a b h}{2}\right\} x^{2} \\
& -\left\{\frac{a(a-b)+a^{2}-b}{2}-\frac{b(a+2 l+2)}{2} h\right\} y^{2} .
\end{aligned}
$$

Now, in view of (18), one can conclude for some positive constant $\alpha$ that

$$
\mathscr{L} V_{1}\left(x_{t}, y_{t}\right) \leq-\alpha\left(x^{2}+y^{2}\right),
$$

provided that

$$
h<\min \left\{\frac{b(a-1)-(l+1) \sigma^{2}}{2 a b}, \frac{a(a-b)+a^{2}-b}{2 b(a+2 l+2)}\right\} .
$$

Since $\int_{-h}^{0} \int_{t+s}^{t} y^{2}(\phi) d \phi d s$ is nonnegative, then we find

$$
V_{1}\left(x_{t}, y_{t}\right) \geq \int_{0}^{y} g(\eta) d \eta+b x^{2}+\frac{1}{2}(y+a x)^{2},
$$

from $g(y) / y \geq a>0$, therefore, we get

$$
V_{1}\left(x_{t}, y_{t}\right) \geq \frac{a}{2} y^{2}+b x^{2}+\frac{1}{2}(y+a x)^{2} .
$$

Then, there exists a positive constant $\beta$ such that

$$
V_{1}\left(x_{t}, y_{t}\right) \geq \beta\left(x^{2}+y^{2}\right) .
$$

Also, since $\left|g^{\prime}(y)\right| \leq l, g(0)=0$, and by using the meanvalue theorem, we get $g(y) \leq l y$. So we can rewrite (14) in the following form:

$$
\begin{aligned}
V_{1}\left(x_{t}, y_{t}\right) \leq & \frac{l}{2} y^{2}+b x^{2}+\frac{1}{2}(y+a x)^{2} \\
& +\gamma \int_{-h}^{0} \int_{t+s}^{t} y^{2}(\phi) d \phi d s .
\end{aligned}
$$
have

It follows that, by using the inequality $2 u v \leq u^{2}+v^{2}$, we

$$
\begin{aligned}
V_{1}\left(x_{t}, y_{t}\right) \leq & \frac{l}{2} y^{2}+b x^{2}+\frac{1}{2} y^{2}+\frac{a}{2}\left(x^{2}+y^{2}\right) \\
& +\frac{a^{2}}{2} x^{2}+\gamma \int_{t-h}^{t}(\theta-t+h) y^{2}(\theta) d \theta .
\end{aligned}
$$


Therefore, we obtain

$$
\begin{aligned}
V_{1}\left(x_{t}, y_{t}\right) & \leq \frac{a^{2}+a+2 b}{2}\|x\|^{2}+\frac{a+l+1}{2}\|y\|^{2}+\frac{\gamma h^{2}}{2}\|y\|^{2} \\
& =\frac{a^{2}+a+2 b}{2}\|x\|^{2}+\frac{a+l+1+\gamma h^{2}}{2}\|y\|^{2} .
\end{aligned}
$$

Then, there exists a positive constant $\nu$ satisfying

$$
V_{1}\left(x_{t}, y_{t}\right) \leq v\left(x^{2}+y^{2}\right)
$$

Thus, from the results (19), (23), and (27), we note that all the conditions of Theorem 4 are satisfied, and then the zero solution of (3) is stochastically asymptotically stable. This completes the proof of Theorem 5 .

In the next section, we shall state and prove our main second result on boundedness of (3) with $p(t, x(t), \dot{x}(t), x(t-$ h)) $\not \equiv 0$.

\section{Further Preliminaries and Boundedness Result}

Consider the $n$-dimensional stochastic delay differential equation (SDDE):

$$
\begin{aligned}
d x(t)= & f(t, x(t), x(t-\tau)) d t \\
& +g(t, x(t), x(t-\tau)) d B(t), \quad t \geq 0,
\end{aligned}
$$

where $f: \mathbb{R}^{+} \times \mathbb{R}^{n} \times \mathbb{R}^{n} \rightarrow \mathbb{R}^{n}$ and $g: \mathbb{R}^{+} \times \mathbb{R}^{n} \times \mathbb{R}^{n} \rightarrow$ $\mathbb{R}^{n \times m}$. In order to solve the equation, we need to know the initial data and we assume that they are given by initial value of $\{x(\theta):-\tau \leq \theta \leq 0\}, x_{0} \in \mathscr{C}\left([-\tau, 0] ; \mathbb{R}^{n}\right)$.

In [23], Liu and Raffoul use Lyapunov second method to determine sufficient conditions for stochastic boundedness of system (28). The theorems in [23] will make significant contribution to the theory of stochastic differential equations, when dealing with equations that might contain unbounded terms.

Definition 6. A solution $x\left(t ; t_{0}, x_{0}\right)$ of (28) is said to be stochastically bounded or bounded in probability, if it satisfies

$$
E^{x_{0}}\left\|x\left(t ; t_{0}, x_{0}\right)\right\| \leq C\left(\left\|x_{0}\right\|, t_{0}\right), \quad \forall t \geq t_{0},
$$

where $E^{x_{0}}$ denotes the expectation operator with respect to the probability law associated with $x_{0}, C: \mathbb{R}^{+} \times \mathbb{R}^{+} \rightarrow$ $\mathbb{R}^{+}$is a constant depending on $t_{0}$ and $x_{0}$. We say that the solutions of (28) are uniformly stochastically bounded, if $C$ is independent of $t_{0}$.

Assumption 7. We assume that for any solution $x(t)$ of (28) and for any fixed $0 \leq t_{0} \leq T<\infty$, the following condition holds:

$$
\begin{array}{r}
E^{x_{0}}\left\{\int_{t_{0}}^{T} V_{x_{i}}^{2}(t, x(t)) g_{i k}^{2}(t, x(t)) d t\right\}<\infty, \\
1 \leq i \leq n, \quad 1 \leq k \leq m .
\end{array}
$$

Assumption 8. A special case of the general condition (30) is the following condition: assume that there exists a function $\chi(t)$ such that

$$
\begin{aligned}
& \left|V_{x_{i}}(t, x(t)) g_{i k}(t, x(t))\right| \leq \chi(t), \\
& x \in \mathbb{R}^{n}, \quad 1 \leq i \leq n, \quad 1 \leq k \leq m,
\end{aligned}
$$

and for any fixed $0 \leq t_{0} \leq T<\infty$,

$$
\int_{t_{0}}^{T} \chi^{2}(t) d t<\infty
$$

Here, we will present the main theorems used in our main boundedness result.

Theorem 9. Assume there exists a function $V(t, x)$ in $\mathscr{C}^{1,2}\left(\mathbb{R}^{+} \times \mathbb{R}^{n} ; \mathbb{R}^{+}\right)$satisfying Assumption 7 , such that for all $(t, x) \in \mathbb{R}^{+} \times \mathbb{R}^{n}$

(i) $\|x\|^{q_{1}} \leq V(t, x) \leq\|x\|^{q_{2}}$,

(ii) $\mathscr{L} V(t, x) \leq-\lambda(t)\|x\|^{r}+\eta(t)$,

(iii) $V(t, x)-V^{r / q_{2}}(t, x) \leq \mu$,

where $\lambda, \eta \in C\left(\mathbb{R}^{+} ; \mathbb{R}^{+}\right), q_{1}, q_{2}$, and $r$ are positive constants, $q_{1} \geq 1$, and $\mu$ is a nonnegative constant. Then, all solutions of (28) satisfy

$$
\begin{aligned}
E^{x_{0}}\left\|x\left(t ; t_{0}, x_{0}\right)\right\| \leq & \left\{V\left(t_{0}, x_{0}\right) e^{-\int_{t_{0}}^{t} \lambda(s) d s}\right. \\
& \left.+\int_{t_{0}}^{t}(\mu \lambda(u)+\eta(u)) e^{-\int_{u}^{t} \lambda(s) d s} d u\right\}^{1 / q_{1}},
\end{aligned}
$$

for all $t \geq t_{0}$.

Theorem 10. Assume there exists a function $V(t, x)$ in $\mathscr{C}^{1,2}\left(\mathbb{R}^{+} \times \mathbb{R}^{n} ; \mathbb{R}^{+}\right)$satisfying Assumption 7 , such that for all $(t, x) \in \mathbb{R}^{+} \times \mathbb{R}^{n}$

(i) $\|x\|^{q_{1}} \leq V(t, x)$,

(ii) $\mathscr{L} V(t, x) \leq-\lambda(t) V^{q_{2}}(t, x)+\eta(t)$,

(iii) $V(t, x)-V^{q_{2}}(t, x) \leq \mu$,

where $\lambda, \eta \in C\left(\mathbb{R}^{+} ; \mathbb{R}^{+}\right), q_{1}$ and $q_{2}$ are two positive constants, $q_{1} \geq 1$, and $\mu$ is a nonnegative constant. Then, all solutions of (28) satisfy

$$
\begin{aligned}
E^{x_{0}}\left\|x\left(t ; t_{0}, x_{0}\right)\right\| \leq\{ & V\left(t_{0}, x_{0}\right) e^{-\int_{t_{0}}^{t} \lambda(s) d s} \\
& \left.+\int_{t_{0}}^{t}(\mu \lambda(u)+\eta(u)) e^{-\int_{u}^{t} \lambda(s) d s} d u\right\}^{1 / q_{1}},
\end{aligned}
$$

for all $t \geq t_{0}$ 
Corollary 11. (1) Assume that the hypotheses of Theorem 9 hold. In addition,

$$
\int_{t_{0}}^{t}\{\mu \lambda(u)+\eta(u)\} e^{-\int_{u}^{t} \lambda(s) d s} d u \leq \mathscr{M}, \quad \forall t \geq t_{0} \geq 0
$$

for some positive constant $\mathscr{M}$; then, all the solutions of (28) are uniformly stochastically bounded.

(2) Assume that the hypotheses of Theorem 10 hold. If condition (35) is satisfied, then all the solutions of (28) are stochastically bounded.

The following theorem is the second main result for (3) with $p \neq \equiv 0$.

Theorem 12. Let the conditions (i) and (ii) of Theorem 5 be satisfied. In addition, we assume that

(iii) $\sigma^{2}<(a b(2 b-1)+b(2 a b-1)) /(3 l+2 b+1)$;

(iv) $|p(t, x(t), \dot{x}(t), x(t-h))| \leq m$, for some $m>0$.

Then, all the solutions of (3) are uniformly stochastically bounded, provided that

$$
\begin{gathered}
h<\min \left\{\frac{a b(2 b-1)+b(2 a b-1)-(3 l+2 b+1) \sigma^{2}}{2 a b(2 b+1)},\right. \\
\left.\frac{3 a(2 a-b)-b}{2 b(6 l+4 b+2 a b+a+2)}\right\} .
\end{gathered}
$$

Proof of Theorem 12. Equation (3) has the following equivalent system:

$$
\begin{aligned}
\dot{x}= & y, \\
\dot{y}= & -g(y)-b x+b \int_{t-h}^{t} y(s) d s \\
& -\sigma x \dot{\omega}(t)+p(t, x, y, x(t-h)) .
\end{aligned}
$$

Consider the Lyapunov functional $V\left(x_{t}, y_{t}\right)$ as

$$
V\left(x_{t}, y_{t}\right)=V_{1}\left(x_{t}, y_{t}\right)+V_{2}\left(x_{t}, y_{t}\right),
$$

where $V_{1}\left(x_{t}, y_{t}\right)$ is defined as (14) and $V_{2}\left(x_{t}, y_{t}\right)$ is defined as

$$
V_{2}\left(x_{t}, y_{t}\right)=a^{2} b x^{2}+2 \int_{0}^{y} g(\eta) d \eta+2 a b x y+b y^{2}+b^{2} x^{2} \text {. }
$$

From (14), (37), and (iv), we find

$$
\begin{aligned}
\mathscr{L} V_{1}\left(x_{t}, y_{t}\right) \leq & -\left\{\frac{b(a-1)}{2}-\frac{l+1}{2} \sigma^{2}-\frac{a b h}{2}\right\} x^{2} \\
& -\left\{\frac{a(a-b)+a^{2}-b}{2}-\left(\frac{b l}{2}+\frac{b}{2}+\gamma\right) h\right\} y^{2} \\
& +\left(\frac{b l}{2}+\frac{a b}{2}+\frac{b}{2}-\gamma\right) \int_{t-h}^{t} y^{2}(s) d s \\
& +a m|x|+(l+1) m|y| .
\end{aligned}
$$

Also, from (39), (37), (i), and (iv), we obtain

$$
\begin{aligned}
\mathscr{L} V_{2}\left(x_{t}, y_{t}\right) \leq & -\left\{a b(2 b-1)-(l+b) \sigma^{2}-a b^{2} h\right\} x^{2} \\
& -\{a(2 a-b)-b(l+b) h\} y^{2} \\
& +\left(b l+b^{2}+a b^{2}\right) \int_{t-h}^{t} y^{2}(s) d s \\
& +2 a b m|x|+2(l+b) m|y| .
\end{aligned}
$$

Therefore, from (38), (40), and (41), we obtain

$$
\begin{aligned}
& \mathscr{L} V\left(x_{t}, y_{t}\right) \\
& \leq-\left\{\frac{a b(2 b-1)+b(2 a b-1)}{2}\right. \\
&\left.-\frac{3 l+2 b+1}{2} \sigma^{2}-\frac{a b(2 b+1)}{2} h\right\} x^{2} \\
&- {\left[\frac{3 a(2 a-b)-b}{2}-\left\{\frac{b(3 l+2 b+1)}{2}+\gamma\right\} h\right] y^{2} } \\
&-\left\{\gamma-\frac{b(3 l+2 b+2 a b+a+1)}{2}\right\} \int_{t-h}^{t} y^{2}(s) d s \\
&+ a m(2 b+1)|x|+(3 l+2 b+1) m|y| .
\end{aligned}
$$

If we take $\gamma=b(3 l+2 b+2 a b+a+1) / 2>0$, we can write (42) in the following form:

$$
\begin{aligned}
& \mathscr{L} V\left(x_{t}, y_{t}\right) \\
& \leq-\left\{\frac{a b(2 b-1)+b(2 a b-1)}{2}\right. \\
&\left.-\frac{3 l+2 b+1}{2} \sigma^{2}-\frac{a b(2 b+1)}{2} h\right\} x^{2} \\
&-\left\{\frac{3 a(2 a-b)-b}{2}-\frac{b(6 l+4 b+2 a b+a+2)}{2} h\right\} y^{2} \\
&+ a m(2 b+1)|x|+(3 l+2 b+1) m|y| .
\end{aligned}
$$

Therefore, if

$$
\begin{gathered}
h<\min \left\{\frac{a b(2 b-1)+b(2 a b-1)-(3 l+2 b+1) \sigma^{2}}{2 a b(2 b+1)},\right. \\
\left.\frac{3 a(2 a-b)-b}{2 b(6 l+4 b+2 a b+a+2)}\right\},
\end{gathered}
$$


then we get

$$
\begin{aligned}
\mathscr{L} V\left(x_{t}, y_{t}\right) \leq & -\vartheta\left(x^{2}+y^{2}\right)+k \vartheta(|x|+|y|) \\
= & -\frac{\vartheta}{2}\left(x^{2}+y^{2}\right) \\
& -\frac{\vartheta}{2}\left\{(|x|-k)^{2}+(|y|-k)^{2}\right\}+\vartheta k^{2} \\
\leq & -\frac{\vartheta}{2}\left(x^{2}+y^{2}\right)+\vartheta k^{2}, \quad \forall k, \vartheta>0 .
\end{aligned}
$$

Thus, condition (ii) of Theorem 9 is satisfied by taking $\lambda(t)=\vartheta / 2, \eta(t)=\vartheta k^{2}$, and $r=2$. Also, we can easily check that conditions (i)-(iii) of Theorem 9 with $q_{1}=q_{2}=2$ and $\mu=0$ are satisfied, using the same techniques which have already been demonstrated in proof of Theorem 5 . With $\lambda(t)=\vartheta / 2, \eta(t)=\vartheta k^{2}$, and $\mu=0$ and using them in (35), we note that

$$
\begin{gathered}
\int_{t_{0}}^{t}\{\mu \lambda(u)+\eta(u)\} e^{-\int_{u}^{t} \lambda(s) d s} d u \\
=9 k^{2} \int_{t_{0}}^{t} e^{-(9 / 2) \int_{u}^{t} d s} d u \leq 2 k^{2}
\end{gathered}
$$

for all $t \geq t_{0} \geq 0$. Thus, condition (35) holds. Now, since

$$
\begin{aligned}
g^{T} & =(0-\sigma x), \\
V_{x} & =\left(V_{1}\right)_{x}+\left(V_{2}\right)_{x} \\
& =2 b x+a y+a^{2} x+2 a^{2} b x+2 a b y+2 b^{2} x, \\
V_{y} & =\left(V_{1}\right)_{y}+\left(V_{2}\right)_{y} \\
& =3 g(y)+a x+y+2 a b x+2 b y .
\end{aligned}
$$

Then, we have

$$
\begin{aligned}
\left|V_{x_{i}}(t, x(t)) g_{i k}(t, x(t))\right| \leq \sigma & \left\{2 a b+a+b+\frac{3 l+1}{2}\right\} x^{2} \\
& \left.+\left\{\frac{3 l+1}{2}+b\right\} y^{2}\right]:=\chi(t) .
\end{aligned}
$$

Hence, the conditions (31) and (32) are satisfied. So by Corollary 11(1), we find that all solutions of (3) are uniformly stochastically bounded and satisfy

$$
E^{x_{0}}\left\|x\left(t ; t_{0}, x_{0}\right)\right\| \leq\left\{C x_{0}^{2}+2 k^{2}\right\}^{1 / 2}, \quad \forall t \geq t_{0} \geq 0,
$$

where $C$ is a constant. This completes the proof of Theorem 12 .

\section{Illustrative Examples}

In this section, we display two examples to illustrate the application of the results we obtained in the previous sections.
Example 1. As an application of Theorem 5, we consider the second-order stochastic delay differential equation

$$
\ddot{x}(t)+4 \dot{x}(t)+\sin \dot{x}(t)+2 x(t-h)+\frac{1}{2} x(t) \dot{\omega}(t)=0 .
$$

The equivalent system of (50) is

$\dot{x}=y$,

$\dot{y}=-(4 y+\sin y)-2 x+2 \int_{t-h}^{t} y(s) d s-\frac{1}{2} x(t) \dot{\omega}(t)$.

From (50), we have

$$
g(y)=4 y+\sin y, \quad b=2, \quad \sigma=\frac{1}{2} .
$$

It is obvious that

$$
\begin{gathered}
\frac{g(y)}{y}=4+\frac{\sin y}{y} \geq 3=a, \quad \forall y, \quad(y \neq 0), \\
\left|g^{\prime}(y)\right|=|4+\cos y| \leq 5=l, \\
\frac{b(a-1)}{2}-\left(\frac{l+1}{2}\right) \sigma^{2}=\frac{10}{8}>0 .
\end{gathered}
$$

Thus,

$$
\begin{aligned}
\mathscr{L} V_{1}\left(x_{t}, y_{t}\right) \leq & -\left(\frac{10}{8}-\frac{6}{2} h\right) x^{2}-\left\{\frac{10}{2}-\left(\frac{12}{2}+\gamma\right) h\right\} y^{2} \\
& +\left(\frac{10}{2}+\frac{6}{2}+\frac{2}{2}-\gamma\right) \int_{t-h}^{t} y^{2}(s) d s .
\end{aligned}
$$

Let us choose $\gamma=b(l+a+1) / 2=9>0$. Then, one can conclude for some positive constant $\alpha$ that

$$
\mathscr{L} V_{1}\left(x_{t}, y_{t}\right) \leq-\alpha\left(x^{2}+y^{2}\right)
$$

provided that $h<\min \{5 / 24,5 / 30\}$.

Since $4+\sin y / y \geq 3=a>0$, therefore we get

$$
V_{1}\left(x_{t}, y_{t}\right) \geq \frac{3}{2} y^{2}+2 x^{2}+\frac{1}{2}(y+3 x)^{2} .
$$

Then, there exists a positive constant $\beta$ such that

$$
V_{1}\left(x_{t}, y_{t}\right) \geq \beta\left(x^{2}+y^{2}\right) \text {. }
$$

Also, by using the fact that $|4+\cos y| \leq 5=l$, we obtain

$$
V_{1}\left(x_{t}, y_{t}\right) \leq \frac{9+3+4}{2}\|x\|^{2}+\frac{1+3+5+9 h^{2}}{2}\|y\|^{2} \text {. }
$$

So there exists a positive constant $\nu$ satisfying

$$
V_{1}\left(x_{t}, y_{t}\right) \leq v\left(x^{2}+y^{2}\right)
$$


Thus, from the results (55), (57), and (59), we note that all the conditions of Theorem 4 are satisfied, so the zero solution of (50) is stochastically asymptotically stable.

Example 2. As an application of Theorem 12, consider the second-order stochastic delay differential equation

$$
\begin{aligned}
& \ddot{x}(t)+4 \dot{x}(t)+\sin \dot{x}(t)+2 x(t-h)+\frac{1}{2} x(t) \dot{\omega}(t) \\
& =p(t, x, \dot{x}, x(t-h)) .
\end{aligned}
$$

The equivalent system of (60)

$$
\begin{gathered}
\dot{x}=y, \\
\dot{y}=-(4 y+\sin y)-2 x+2 \int_{t-h}^{t} y(s) d s \\
-\frac{1}{2} x(t) \dot{\omega}(t)+p .
\end{gathered}
$$

From (60), we have

$$
g(y)=4 y+\sin y, \quad b=2, \quad \sigma=\frac{1}{2} .
$$

It is obvious that

$$
\begin{gathered}
\frac{g(y)}{y}=4+\frac{\sin y}{y} \geq 3=a, \quad \forall y, \quad(y \neq 0), \\
\left|g^{\prime}(y)\right|=|4+\cos y| \leq 5=l, \\
\frac{a b(2 b-1)+b(2 a b-1)}{3 l+2 b+1}=2>\sigma^{2}=\frac{1}{4}, \\
m=0.02 .
\end{gathered}
$$

Thus,

$$
\begin{aligned}
\mathscr{L} V\left(x_{t}, y_{t}\right) \leq & -\left(\frac{35}{2}-\frac{30}{2} h\right) x^{2}-\left(\frac{34}{2}-\frac{110}{2} h\right) y^{2} \\
& +0.3|x|+0.4|y| .
\end{aligned}
$$

Since $\gamma=b(3 l+2 b+2 a b+a+1) / 2=35>0$, provided that $h<\min \{35 / 60,34 / 220\}$.

If we take $\vartheta=8.5$ and $k=0.047$. Thus, condition (ii) of Theorem 9 is satisfied by taking $\lambda(t)=4.25, \eta(t)=0.019$, and $r=2$. Also, we can easily check that the conditions (i)-(iii) of Theorem 9 with $q_{1}=q_{2}=r=2$ and $\mu=0$ are satisfied, since

$$
\int_{t_{0}}^{t}\{\mu \lambda(u)+\eta(u)\} e^{-\int_{u}^{t} \lambda(s) d s} d u \leq 0.0044
$$

for all $t \geq t_{0} \geq 0$. Thus, condition (35) holds. Now since

$$
\left|V_{x_{i}}(t, x(t)) g_{i k}(t, x(t))\right| \leq \frac{1}{2}\left[25 x^{2}+10 y^{2}\right]:=\chi(t),
$$

hence the conditions (31) and (32) are satisfied. So by Corollary 11, all solutions of (60) with $|p| \leq 0.02$ are uniformly stochastically bounded and satisfy

$$
E^{x_{0}}\left\|x\left(t ; t_{0}, x_{0}\right)\right\| \leq\left\{x_{0}^{2}+0.0044\right\}^{1 / 2}, \quad \forall t \geq t_{0} \geq 0 .
$$

\section{Conflict of Interests}

The authors declare that there is no conflict of interests regarding the publication of this paper.

\section{References}

[1] L. Arnold, Stochastic Differential Equations: Theory and Applications, John Wiley \& Sons, New York, NY, USA, 1974.

[2] A. Friedman, Stochastic Differential Equations and Their Applications, Academic Press, 1976.

[3] I. I. Gikhman and A. V. Skorokhod, Stochastic Differential Equations, Springer, Berlin, Germany, 1972.

[4] J. K. Hale and S. M. V. Lunel, Introduction to Functional Differential Equations, Springer, New York, NY, USA, 1993.

[5] R. Z. Khasminskii, Stochastic Stability of Differential Equations, Sijthoff \& Noordhoff, Germantown, Md, USA, 1980.

[6] V. B. Kolmanovskii and A. D. Myshkis, Introduction to the Theory and Applications of Functional Differential Equations, Kluwer Academic Publishers, Dordrecht, The Netherlands, 1999.

[7] V. Kolmanovskii and L. Shaikhet, "Construction of Lyapunov functionals for stochastic hereditary systems: a survey of some recent results," Mathematical and Computer Modelling, vol. 36, no. 6, pp. 691-716, 2002.

[8] V. Kolmanovskii and L. Shaikhet, "About some features of general method of Lyapunov functionals construction," Stability and Control: Theory and Applications, vol. 6, no. 1, pp. 49-76, 2004.

[9] S.-E. A. Mohammed, Stochastic Functional Differential Equations, vol. 99 of Research Notes in Mathematics, Pitman Publishing, 1984.

[10] B. Øksendal, Stochastic Differential Equations: An Introduction with Applications, Springer, New York, NY, USA, 2000.

[11] R. Reissing, E. Sansone, and R. Conti, Non-Linear Differential Equations of Higher-Order, Noordhoff International Publishing, Leyden, Mass, USA, 1974.

[12] U. H. Thygesen, "A survey of Lyapunov techniques for stochastic differential equations," IMM Technical Report, 1997.

[13] C. Yuan and X. Mao, "Asymptotic stability and boundedness of stochastic differential equations with respect to semimartingales," Stochastic Analysis and Applications, vol. 21, no. 3, pp. 737-751, 2003.

[14] X. Mao and L. Shaikhet, "Delay-dependent stability criteria for stochastic differential delay equations with Markovian switching," Stability and Control: Theory and Applications, vol. 3, no. 2, pp. 88-101, 2000.

[15] X. Mao, "Some contributions to stochastic asymptotic stability and boundedness via multiple Lyapunov functions," Journal of Mathematical Analysis and Applications, vol. 260, no. 2, pp. 325340, 2001.

[16] R. Rezaeyan and R. Farnoosh, "Stochastic differential equations and application of the Kalman-Bucy filter in the modeling of RC circuit," Applied Mathematical Sciences, vol. 4, no. 23, pp. 1119$1127,2010$.

[17] A. Rodkina and M. Basin, "On delay-dependent stability for a class of nonlinear stochastic delay-differential equations," Mathematics of Control, Signals, and Systems, vol. 18, no. 2, pp. 187-197, 2006. 
[18] S. Yi, J. Ming-Hui, and L. Xiao-Xin, "Asymptotic stabilities of stochaastic functional differential equations," Applied Mathematics and Mechanics (English Edition), vol. 27, no. 11, pp. 15771584, 2006.

[19] E. Kolarova, "An application of stochastic integral equations to electrical networks," Acta Electotechnica et Informatica, vol. 8, no. 3, pp. 14-17, 2008.

[20] D. Y. Xu, B. Li, S. J. Long, and L. Y. Teng, "Corrigendum to 'moment estimate and existence for solutions of stochastic functional differential equations' [Nonlinear Anal.: TMA 108 (2014) 128-143]," Nonlinear Analysis: Theory, Methods \& Applications, vol. 114, pp. 40-41, 2015.

[21] X. Mao, Stochastic Differential Equations and Their Applications, Horwood Publishing, Chichester, UK, 1997.

[22] L. Y. Teng, S. J. Long, and D. Y. Xu, "On solvability of neutral stochastic functional differential equations with infinite delay," Communications on Pure and Applied Analysis, vol. 18, pp. 325344, 2014.

[23] R. Liu and Y. Raffoul, "Boundedness and exponential stability of highly nonlinear stochastic differential equations," Electronic Journal of Differential Equations, vol. 143, pp. 1-10, 2009. 


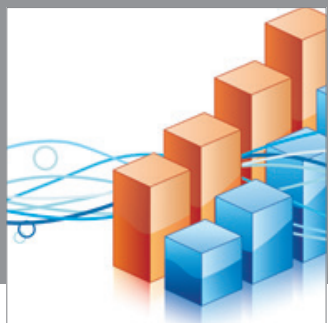

Advances in

Operations Research

mansans

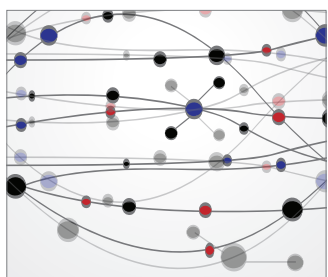

The Scientific World Journal
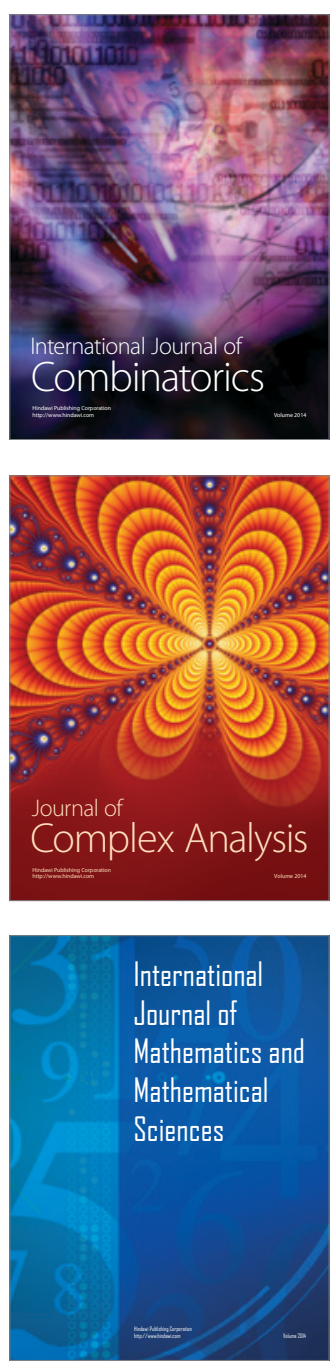
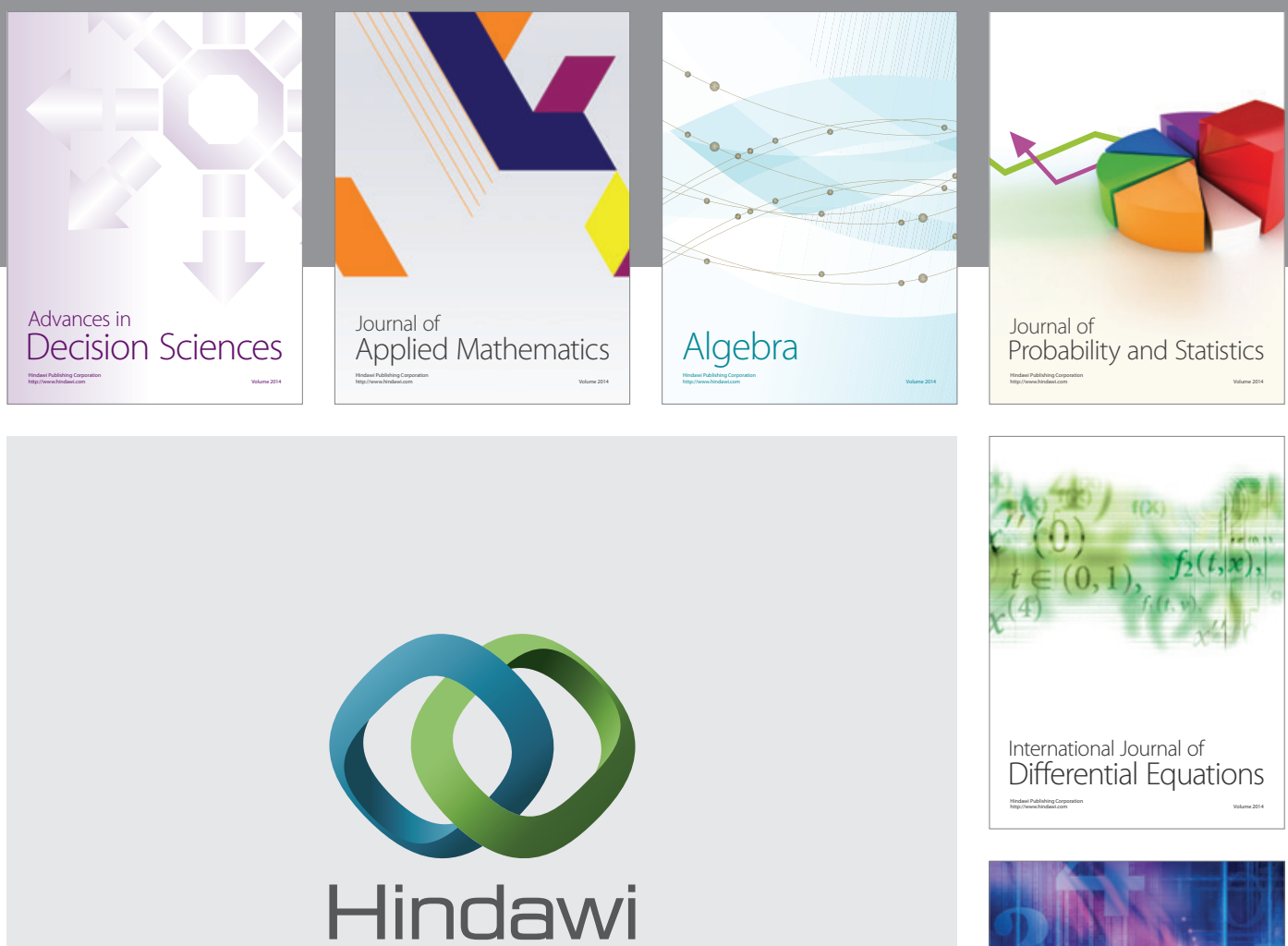

Submit your manuscripts at http://www.hindawi.com
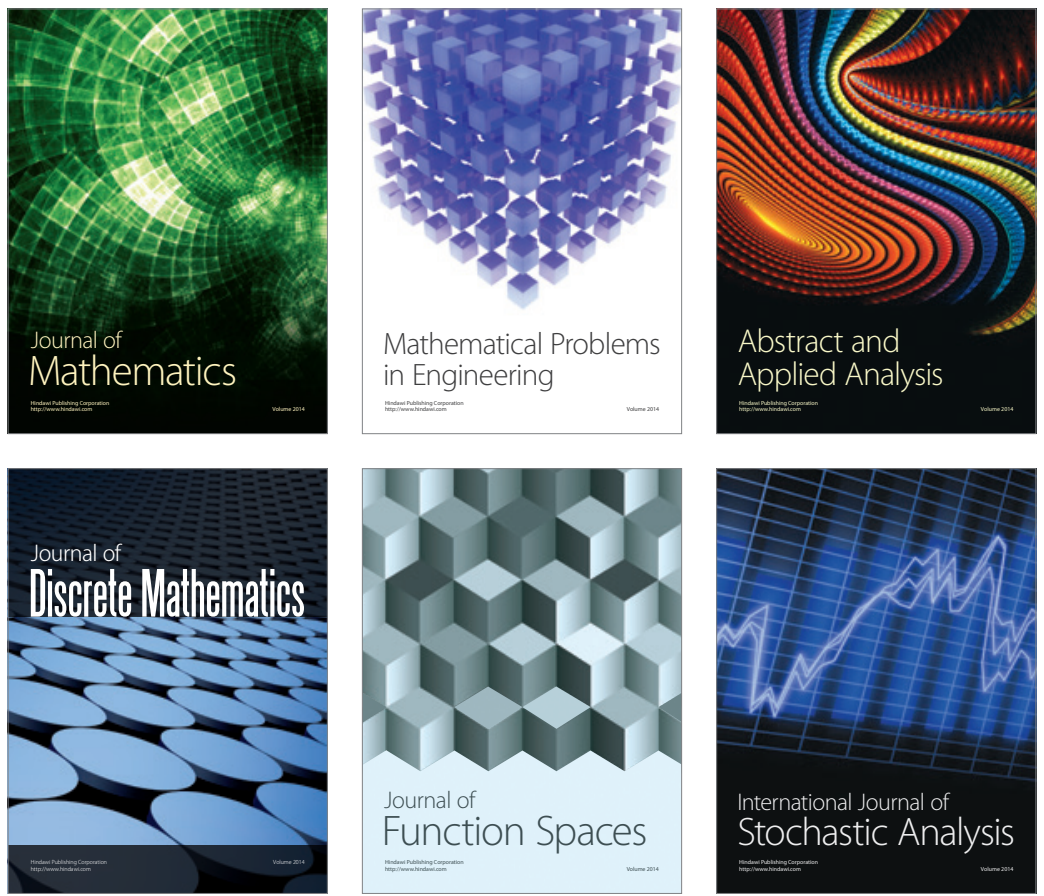

Journal of

Function Spaces

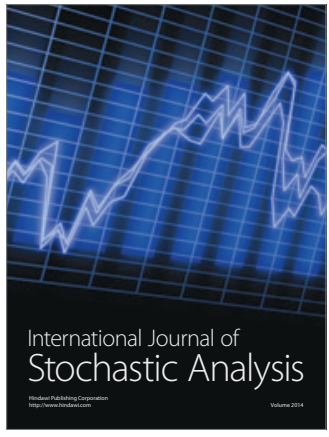

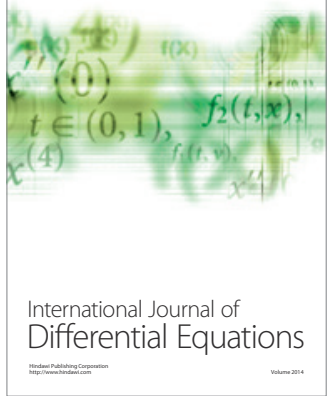
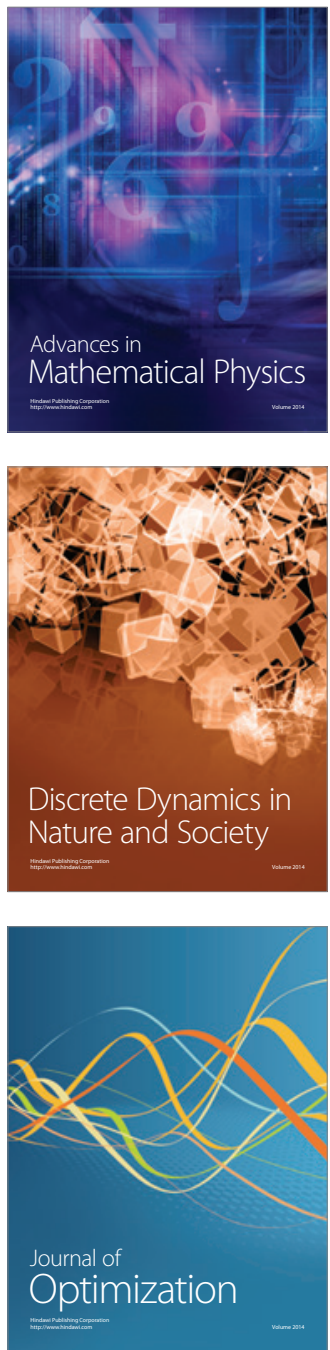